\title{
Quasi-intrinsic semiconducting state of titanyl-phthalocyanine films obtained under ultrahigh vacuum conditions
}

\author{
AUTHOR(S): \\ Tada, H; Touda, H; Takada, M; Matsushige, K
}

\section{CITATION:}

Tada, $H$... [et al]. Quasi-intrinsic semiconducting state of titanyl-phthalocyanine films obtained under ultrahigh vacuum conditions. APPLIED PHYSICS LETTERS 2000, 76(7): 873-875

ISSUE DATE:

2000-02-14

URL:

http://hdl.handle.net/2433/39635

\section{RIGHT:}

Copyright 2000 American Institute of Physics. This article may be downloaded for personal use only. Any other use requires prior permission of the author and the American Institute of Physics. 


\title{
Quasi-intrinsic semiconducting state of titanyl-phthalocyanine films obtained under ultrahigh vacuum conditions
}

\author{
Hirokazu Tada, ${ }^{a}$ Hiroshi Touda, Masaki Takada, and Kazumi Matsushige \\ Department of Electronic Science and Engineering, Kyoto University, Sakyo-ku, Kyoto 606-8501, Japan
}

(Received 13 September 1999; accepted for publication 16 December 1999)

\begin{abstract}
We investigated electrical properties of titanyl-phthalocyanine (TiOPc) films under ultrahigh vacuum (UHV) conditions to avoid the influence of gas adsorption. The field-effect measurement revealed that TiOPc films exhibited an $n$-type semiconducting behavior in UHV. The electron mobility at room temperature was $9 \times 10^{-6} \mathrm{~cm}^{2} \mathrm{~V}^{-1} \mathrm{~s}^{-1}$ with activation energy of $0.20 \mathrm{eV}$. The conductivity and carrier density were $9 \times 10^{-8} \mathrm{~S} \mathrm{~cm}^{-1}$ and $6 \times 10^{16} \mathrm{~cm}^{-3}$, respectively. A clear conversion from $n$-type to $p$-type behavior was observed when the film was exposed to oxygen. Strict control of atmosphere made it possible to obtain a quasi-intrinsic state where both $p$ - and $n$-type conductions appeared simultaneously. (C) 2000 American Institute of Physics.

[S0003-6951(00)01507-2]
\end{abstract}

Organic semiconductors have recently been used as active layers in electronic devices such as field-effect transistors (FETs) ${ }^{1-4}$ and electroluminescent diodes (LEDs). ${ }^{5}$ The mechanism of carrier transport in organic semiconductors becomes one of the most important subjects to be elucidated for improvements of devices. The electrical properties of organic materials are, however, modified easily by adsorbed gas molecules, which makes it difficult to obtain reliable experimental results. Most organic semiconductors show a $p$-type behavior due to unavoidable adsorption of oxygen molecules which act as electron acceptors in the semiconductors. It is also due to the adsorption of oxygen that $p / n$ conduction types cannot be controlled easily by doping of chemical substances, which causes a disadvantage in organic devices. It is thus essential to strip away the veil of oxygen and to reveal the intrinsic electrical properties of organic semiconductors.

In the present work, we have investigated carrier transport of phthalocyanine films by preparing FETs under ultrahigh vacuum (UHV) conditions. Preparation and characterization of the specimen were carried out in the UHV system to avoid the influence of oxygen.

FET has been widely used to evaluate the carrier mobility of organic semiconductors. ${ }^{1-4,6-8}$ A highly doped $n^{+}$-Si(111) plate was used as the gate electrode on which the $\mathrm{SiO}_{2}$ layer with the thickness of about $300 \mathrm{~nm}$ was formed by thermal oxidation. The interdigital $\mathrm{Au}(20 \mathrm{~nm}) / \mathrm{Cr}$ $(10 \mathrm{~nm})$ electrodes, which consisted of 25 pairs with $25 \mu \mathrm{m}$ in spacing and $6 \mathrm{~mm}$ in width, were prepared on the surface and used as source and drain electrodes. The equipment consisted of two vacuum chambers as shown elsewhere: ${ }^{8}$ one was used for film growth and the other was for electrical measurement. The base pressures of both chambers were about $1 \times 10^{-6} \mathrm{~Pa}$. The partial pressures of residual gas molecules were measured with a quadrupole mass filter. The pressures of hydrogen, water and nitrogen were about 5 $\times 10^{-7} \mathrm{~Pa}$, and that of oxygen was about $3 \times 10^{-9} \mathrm{~Pa}$.

${ }^{a}$ Electronic mail: tada@kuee.kyoto-u.ac.jp
The TiOPc powder purchased from Aldrich was purified by vacuum sublimation and charged into a Knudsen cell. The substrate was kept at room temperature during film growth in the present experiment. The thickness of the films was about $40 \mathrm{~nm}$. The specimens were transferred into the measurement chamber without being exposed to the air, where FET characteristics were measured under UHV and in various gas atmospheres.

The mobility was calculated by fitting the curves observed to the following equation available for metal-oxidesemiconductor FET (MOSFET) ${ }^{1,9}$ in the saturation region:

$$
I_{D}(\mathrm{sat})=\mu W C\left(V_{G}-V_{T}\right)^{2} / 2 L,
$$

and in the linear region:

$$
I_{D}=\mu W C\left(V_{G}-V_{T}\right) V_{D} / L .
$$

Here, $I_{D}$ (sat) is the (saturated) drain current, $\mu$ is the mobility, $V_{G}$ and $V_{D}$ are the voltages applied to the gate and drain electrodes, respectively, $V_{T}$ is the threshold voltage, $L, W$, and $C$ are the channel length, channel width, and capacitance of the gate insulator, respectively. The activation energy of carrier transport was measured by an Arrhenius plot of $\mu$.

The dark conductivity was measured for TiOPc films prepared on glass substrates which had interdigital gold electrodes with a gap width of $100 \mu \mathrm{m}$. The carrier density, $N$, was estimated by the following equation using the obtained values of dark conductivity, $\sigma$, and carrier mobility, $\mu$ :

$$
N=\sigma / e \mu \text {. }
$$

Figures 1(a) and 1(b) show FET characteristics of a TiOPc film measured in vacuum with the electric circuits shown in the insets. When the positive biases were applied to the gate electrode, the drain current increased as shown in Fig. 1(b). The electrons were accumulated near the interface between $\mathrm{SiO}_{2}$ and the TiOPc film. On the contrary, a depletion of electrons occurred when the gate electrode was biased positively, as shown in Fig. 1(a). These results indicate that the TiOPc film has an $n$-type semiconducting behavior in vacuum. The hole conduction did not occur when the gate voltage was increased up to $100 \mathrm{~V}$. The field-effect electron 

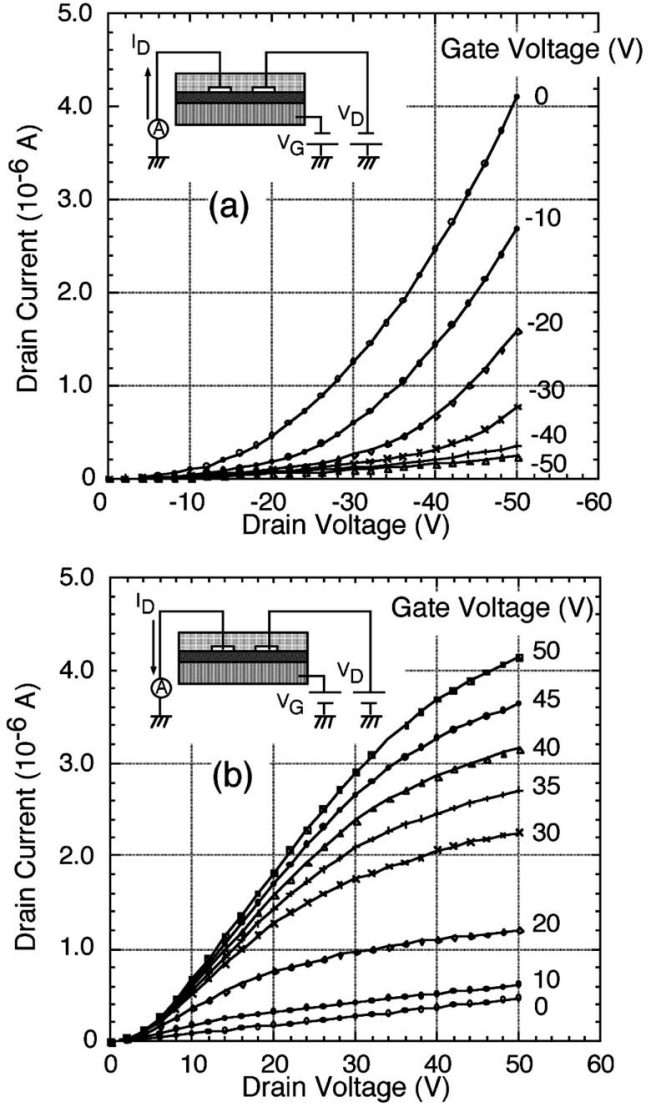

FIG. 1. FET characteristics of a TiOPc film measured in vacuum without being exposed to air. Electric circuits used are shown in the figures: (a) $p$-type circuit and (b) $n$-type circuit.

mobility was evaluated to be $9 \times 10^{-6} \mathrm{~cm}^{2} / \mathrm{V}$ s of TiOPc films at room temperature. This is almost the same value as the hole mobility for a TiOPc film shown below. Temperature dependence of the electron mobility is shown in Fig. 2 with the temperature range between 300 and $400 \mathrm{~K}$. The carrier transport was found to follow a thermal activated hopping mechanism with activation energy of $0.20 \mathrm{eV}$. The origin of the decrease in mobility observed above $350 \mathrm{~K}$ is uncertain. The temperature dependence of electric conductivity of a film deposited on glass was also measured. The conductivity at room temperature was $9 \times 10^{-8} \mathrm{~S} \mathrm{~cm}^{-1}$ with activation energy of $0.35 \mathrm{eV}$. The conductivity observed is

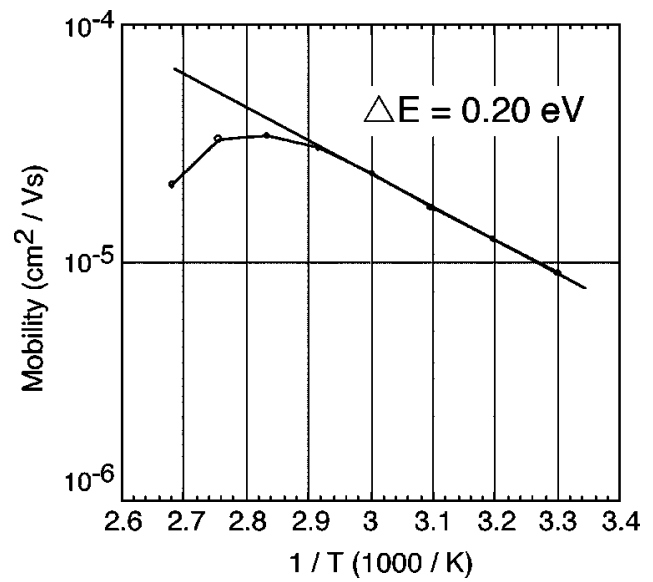
FIG. 2. Arrhenius plot of electron mobility. the films at this stage. Figures 4(a) and 4(b) show FET char-
Downloaded 30 May 2007 to 130.54 .110 .22 . Redistribution subject to AIP license or copyright, see http://apl.aip.org/apl/copyright.jsp
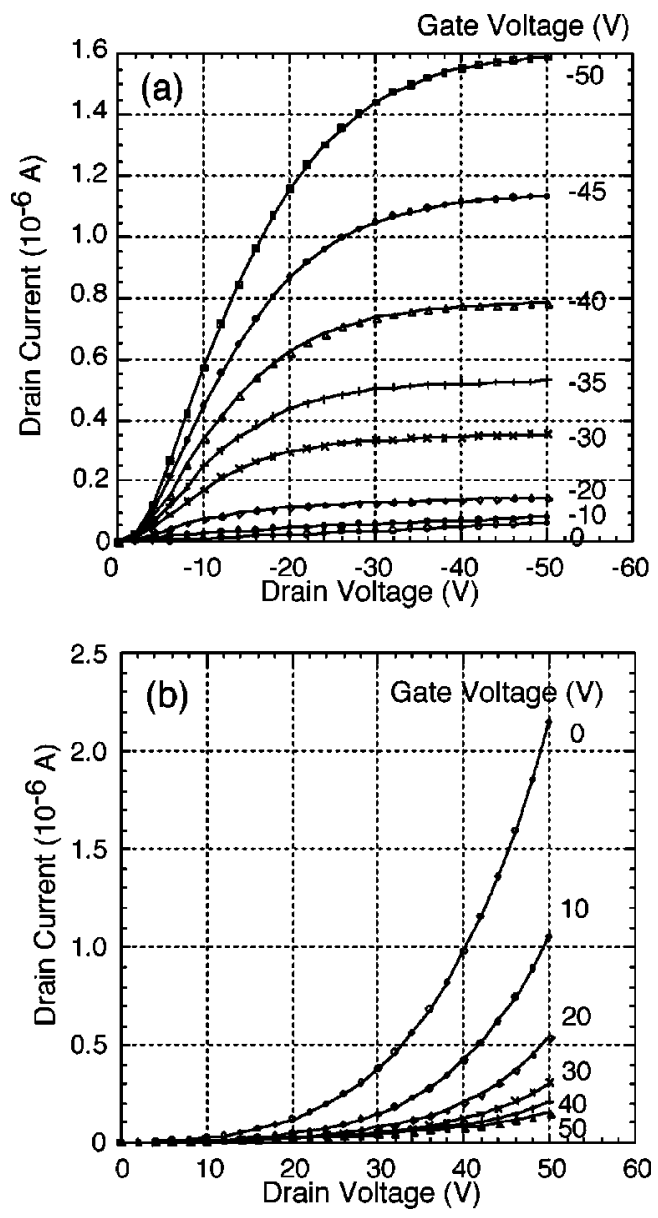

FIG. 3. FET characteristics of a TiOPc film obtained after oxygen exposure with (a) a $p$-type circuit and (b) an $n$-type circuit.

rather high comparing to the values reported for ordinal Pc films. ${ }^{7,10}$ The high conductivity is attributed to high carrier density of $6 \times 10^{16} \mathrm{~cm}^{-3}$. It was reported that lutetium and thulium bisphthalocyaniens also showed an $n$-type semiconducting behavior in vacuum with the charge carrier density as high as $10^{18} \mathrm{~cm}^{-3}{ }^{6}$ The high carrier density observed indicates the existence of impurities which act as electron donors in the films. As one possibility, contamination by residual water in the UHV chamber is considered. There is another possibility of contamination by chemical substances during synthesis, which cannot be removed easily by vacuum sublimation. Further investigation is currently under way to clarify the origin of electron conduction.

Electron conduction of the films disappeared immediately when the oxygen gas was introduced into the chamber. Figures 3(a) and 3(b) show FET characteristics of the film observed in an oxygen atmosphere. The film is found to exhibit a $p$-type semiconducting behavior. The hole mobility at room temperature was $1 \times 10^{-5} \mathrm{~cm}^{2} \mathrm{~V}^{-1} \mathrm{~s}^{-1}$ with activation energy of $0.13 \mathrm{eV}$. The electric conductivity decreased first on exposure to oxygen. It had a minimum value and increased gradually. The minimum value observed in the present experiment was about $10^{-12} \mathrm{~S} \mathrm{~cm}^{-1}$ at room temperature, where the electrons were compensated by almost the same number of holes generated through adsorption of oxygen molecules.

It is interesting to investigate the FET characteristics of the films at this stage. Figures $4(\mathrm{a})$ and 4(b) show FET char-
AIP license or copyright, see http://apl.aip.org/apl/copyright.jsp 

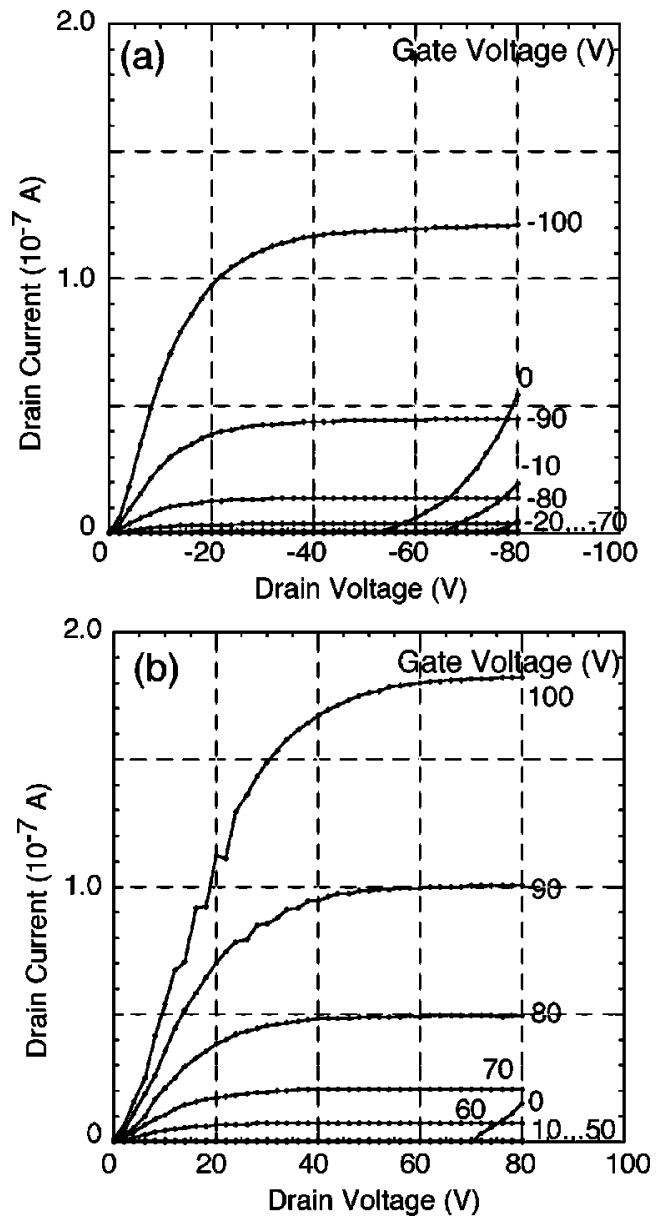

FIG. 4. FET characteristics of a TiOPc film measured in a well-controlled atmosphere. Both (a) $p$-type and (b) $n$-type conductions appeared simultaneously.

acteristics of a TiOPc film observed just after introducing small amount of oxygen. The film exhibits an intrinsic behavior where both $p$ - and $n$-type conductions appeared simultaneously. High electric field was required to accumulate the carriers, since the carrier densities were very small. The current measured at low gate voltage increases rapidly with the drain voltage, which indicates that the conduction was dominated by the space-charge limited process characteristic of insulators. The hole and electron mobilities were evaluated to be $2.0 \times 10^{-6}$ and $2.7 \times 10^{-6} \mathrm{~cm}^{2} \mathrm{~V}^{-1} \mathrm{~s}^{-1}$, respectively. The carrier density estimated using the minimum value of the electric conductivity observed, $10^{-12} \mathrm{~S} \mathrm{~cm}^{-1}$, was about $10^{12} \mathrm{~cm}^{-3}$ both for positive and negative carriers. This value can be regarded as intrinsic carrier density, although the value is larger by more than two orders of magnitude than the value estimated using the band gap energies of $1.1-1.7 \mathrm{eV}$ for Pc compounds. ${ }^{9,10}$ This may be attributed to heavy effective mass of carriers of organic semiconductors.

The $n$-type conduction disappeared immediately after oxygen exposure while the $p$-type conduction disappeared in an $\mathrm{NH}_{3}$ atmosphere. This result demonstrates the possibility of $p / n$ conduction control of organic semiconductors by doping of stable chemical substances to freshly prepared films in UHV.

This work was partly supported by a Grant-in-Aid for Scientific Research on Priority Areas-Electrochemistry of Ordered Interfaces, from the Ministry of Education, Science, Sports and Culture of Japan (No. 10131237). One of the authors (H.T.) is grateful to Murata Foundation for the grant of this work.

${ }^{1}$ G. Horowitz, Adv. Mater. 10, 365 (1998), and references therein.

${ }^{2}$ H. E. Katz, J. Mater. Chem. 7, 369 (1997).

${ }^{3}$ D. J. Gundlach, Y. Y. Lin, T. N. Jackson, S. F. Nelson, and D. G. Schlom, IEEE Electron Device Lett. 18, 87 (1997).

${ }^{4}$ Z. Bao, A. J. Lovinger, and J. Brown, J. Am. Chem. Soc. 120, 207 (1998).

${ }^{5}$ R. H. Friend, R. W. Gymer, A. B. Holmes, J. H. Burroughes, R. N. Marks, C. Taliani, D. D. C. Bradley, D. A. Dos Santos, J. L. Brédas, M. Lögdlund, and W. R. Salaneck, Nature (London) 397, 121 (1999), and references therein.

${ }^{6}$ G. Guillaud, M. Sadoun, M. Maitrot, J. Simon, and M. Bouvet, Chem. Phys. Lett. 167, 503 (1990).

${ }^{7}$ K. Kudo, T. Sumimmoto, K. Hiraga, S. Kuniyoshi, and K. Tanaka, Jpn. J. Appl. Phys., Part 1 36, 6994 (1998).

${ }^{8}$ H. Tada, H. Touda, M. Takada, and K. Matsushige, J. Porphirins and Phthalocyanines 3, 667 (1999).

${ }^{9}$ S. M. Sze, Physics of Semiconductor Devices (Wiley, New York, 1981), Chap. 8.

${ }^{10}$ D. Wöhrle, L. Kreienhoop, and D. Schlettwein, in Phthalocyanines, edited by C. C. Leznoff and A. B. P. Lever (VCH, New York, 1996), Vol. 4, Chap. 6. 